\title{
The Quantum-Mechanical Theory of THz Radiation Conical Structure Emitted from Extended Gas Media
}

\author{
S.Yu. Stremoukhov ${ }^{1,2 a}$ and A.V. Andreev ${ }^{1}$ \\ ${ }^{1}$ Faculty of Physics M.V. Lomonosov Moscow State University, 119991 Leninskie Gory, Moscow, Russia, \\ ${ }^{2}$ National Research Center "Kurchatov Institute", 123182 pl. Akademika Kurchatova, 1, Moscow, Russia
}

\begin{abstract}
We present the quantum-mechanical description of the $\mathrm{THz}$ amplitude periodical variation appearing due to dispersion effects and affecting the angular spectra of $\mathrm{THz}$ emission from extended argon gas interacting with a two-color laser field.
\end{abstract}

Here we report the results of studying the specific features of the $\mathrm{THz}$ emission in the extended medium irradiated by a two-color laser field. The amplitude of the $\mathrm{THz}$ field depends on the phase difference between the components of a two-color field [4]. As a result, the intensity of the $\mathrm{THz}$ emission oscillates along with the delay time between the fundamental $(\omega)$ and the second harmonic $(2 \omega)$ pulses. Due to dispersion effects, the phase difference between the components of the two-color laser field will vary along with the traveled distance in an extended medium. Hence, the frequencyangular spectra of the $\mathrm{THz}$ emission should be dependent on the geometry of radiating volume, but the consistent analysis of the spatial distribution of the $\mathrm{THz}$ radiation emitted in the extended medium has not been done yet. Moreover, due to the strong dependency of the THz generation efficiency on the relative phase of $\omega$ and $2 \omega$ pulses this phenomenon is under active study [5]. At the same time, widely used theories [1, 2] predict a different dependency. For example, in [1] it is predicted that the maximum of the $\mathrm{THz}$ intensity is achieved when the fields at the fundamental frequency and the second harmonic have the same phase (the relative phase is equal to zero), while the authors of [2] suppose that the maximum is when the relative phase between the pulses is equal to $\pi / 2$. Other researches predict some other results [5].

In order to investigate the specific features of the $\mathrm{THz}$ radiation generation as a function of the delay time between $\omega$ and $2 \omega$ pulses (as a function of the relative phase) we have used the nonperturbative theory of a single atom interaction with a laser field [6]. It is based on the usage of the basis of "an atom in the external field" eigenfunctions when transforming the Schrodinger equation into the set of differential equations for the probability amplitudes of atomic states and when simultaneosly transforming the atomic current density. Despite of the fact that the theory can be used to describe such kind of interactions in non-perturbative regime, we have applied it to the description of a single atom interaction with a two-color laser field in ionization-free regime, i.e. when we limit the maximal amplitude of the laser field intensity and take into account 13 discrete "an atom in the field" wavefunctions (the detailed description of the applied model is given in [3]).

Using the model described in [3] we have calculated the dependence of the $\mathrm{THz}$ radiation generated from a single Ar atom interacting with co-polarized $\omega$ and $2 \omega$ pulses of Ti:Sa laser (the

${ }^{\text {a }}$ Corresponding author: sustrem@gmail.com

This is an Open Access article distributed under the terms of the Creative Commons Attribution License 4.0, which permits unrestricted use, distribution, and reproduction in any medium, provided the original work is properly cited. 
parameters of the laser field have been chosen for the scheme presented in [7]). The following dependencies calculated for the maximum of the generated photoemission spectra $(\approx 11 \mathrm{THz})$ and for the radiation at $1 \mathrm{THz}$ are presented in Fig.1(a). It is clearly seen that the dependencies show the oscillatory behavior with a period of $\approx 660$ as which is equal to a half of the period of the second harmonic oscillations for the Ti:Sa laser. The dependencies on the delay time can be easily recalculated to the dependency on the relative phase between the pulses [7] with a maximum at $\pi \cdot(2 \mathrm{n}+$ 1)/2 ( $\mathrm{n}$ being the integer). Using two different seed lasers (Ti:Sa and $\mathrm{Cr}: \mathrm{F}$ ), two different atomic gases (Ar and $\mathrm{Ne}$ ) and two different angles between the polarizations of the two-color laser field components $(0$ and $\pi / 2)$ we have numerically demonstrated that the period of the $\mathrm{THz}$ intensity oscillations is equal to a half of the period of the second harmonic oscillations and the value of phase of the $\mathrm{THz}$ signal maximum is equal to $\pi \cdot(2 \mathrm{n}+1) / 2$ (n being the integer) which is confirmed by a number of investigations (see, e.g., [2]).
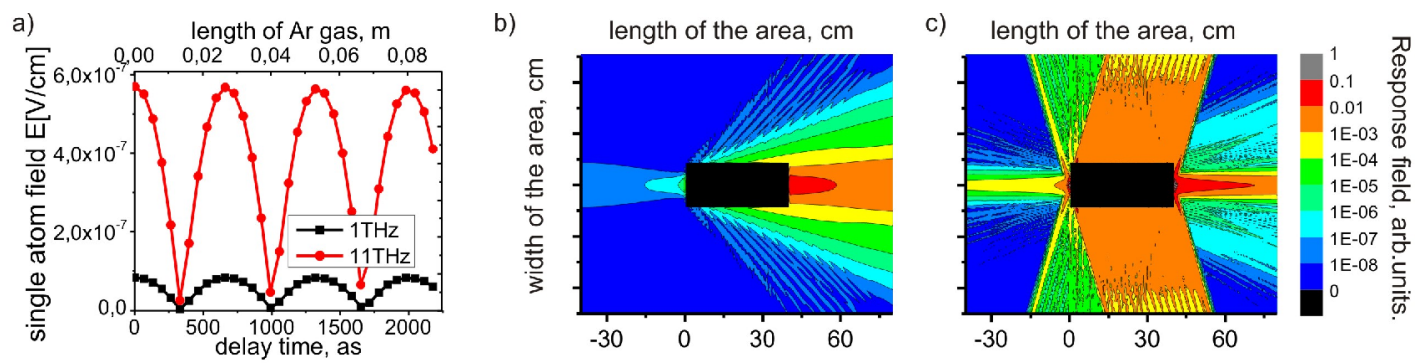

Figure 1. (a) The THz field at a frequency of $11 \mathrm{THz}$ (a curve with circles) and $1 \mathrm{THz}$ (a curve with squares) as a function of the delay time (a lower axis) and the length of Ar gas (an upper axis); the radiation at $1 \mathrm{THz}$ (b) and 10 $\mathrm{THz}$ (c) emitted by the gas cells $40 \mathrm{~cm}$ long and $1.8 \mathrm{~cm}$ wide. The black rectangle shows the volume of the gas cell.

The delay time between the pulses increases along with the position in the gas due to dispersion. As a result the temporal oscillations of the THz efficiency can be transformed into spatial oscillations of the value along with traveled coordinate with a period $\approx 2.7 \mathrm{~cm}$ (for argon gas, see Fig.1 (a) an upper axis). Thus, the position of an atom inside the gas affects the value of the $\mathrm{THz}$ radiation field generated by the atom.

The angular-frequency spectra of the $\mathrm{THz}$ emission are presented in figure 1 (b) and (c) for the frequencies of 1 and $10 \mathrm{THz}$ respectively, calculated for the gas cells $40 \mathrm{~cm}$ long and $1.8 \mathrm{~cm}$ wide. One can see that the angular spectrum of emission looks like a series of nested cones. Such kind of angular spectra is due to the axial symmetry of emitting medium and a finite size of the illuminating laser beam. Fig. 1 (b) apparently demonstrates that the emission at $1 \mathrm{THz}$ has pure cone structure which coincides with a previous study of the THz generation (see, e.g., [8]). At the same time the high frequency emission at $10 \mathrm{THz}$ (Fig. 1 (c)) along with the cone structure has additional sideband lobes. Our additional calculations show that the amplitude of the $\mathrm{THz}$ signal and the apex angle nonlinearly depend on the length and the width of the gas cell.

We have investigated the origin of the sideband lobes and have shown that the sideband lobes appeared only due to the spatial oscillations of macroscopic atomic current.

\section{References}

1. D. J. Cook, R. M. Hochstrasser, Opt. Lett., 25, 1210 (2000)

2. K.-Y. Kim, J. H. Glownia, et.al., Opt. Expr., 15, 4577 (2007)

3. A.V. Andreev, S.Yu. Stremoukhov, Phys. Rev. A, 87, 053416 (2013)

4. J. Dai, X.-C. Zhang, Appl. Phys. Lett., 94, 021117 (2009)

5. H. Dai, J. Liu, J. Opt., 13, 055201 (2011)

6. A.V. Andreev, S.Yu. Stremoukhov, O.A. Shoutova, Eur. Phys. Journ. D 66:16 (2012)

7. S.Yu. Stremoukhov, A.V. Andreev, Laser Phys. Lett., 12, 015402 (2015)

8. H. Zhong, N. Karpowicz, X.-C. Zhang, Appl. Phys. Lett., 88, 261103 (2006) 